\title{
EVALUACIÓN DE LOS MÉTODOS DE MUESTREO PROBABILÍSTICOS EN LA CARACTERIZACIÓN DE SITIOS ARQUEOLÓGICOS POR MEDIO DE SONDEOS SUBSUPERFICIALES
}

\author{
EVALUATION OF PROBABILISTIC SAMPLING METHODS IN THE \\ CHARACTERIZATION OF ARCHAEOLOGICAL SITES THROUGH SUBSURFACE \\ SOUNDING
}

\author{
Luis E. Cornejo B. ${ }^{1}$
}

\begin{abstract}
Se presentan los resultados de una evaluación de los métodos de muestreo probabilísticos aplicados al sondeo subsuperficial (p.ej., pozos de sondeo, barrenos, etc.) como herramientas para evaluar la distribución de densidad de restos arqueológicos subsuperficiales. Por medio de la simulación de tres tipos de sitios con distribuciones de densidad de materiales arqueológicos diferentes y de la aplicación sobre ellos de distintos esquemas y fracciones de muestreo, se observa el grado de error en que se representa la distribución de densidad de materiales lograda con distintas alternativas de muestreo probabilístico. Se concluye, por un lado, que un esquema de muestreo escasamente utilizado en arqueología de campo, el muestreo por transectos intervalares, produce menos errores. Por otro lado, más allá del resultado esperable sobre la relación entre el tamaño de la muestra y la precisión con que se representa un universo, ha sido posible estimar cual rango de fracción de muestreo produce errores aceptables.
\end{abstract}

Palabras claves: evaluación subsuperficial, esquemas de muestreo, fracción de la muestra.

This paper presents the results of an evaluation of the probabilistic sampling methods applied to subsurface sounding (e.g. test pit, auger holes) as tools to measure the density distribution of subsurface archaeological remains. By means of the simulation of three types of sites with different density distributions of subsurface archaeological materials and the application of different sampling schemes and sampling fractions, the degree of error in representing the density distribution of subsurface materials achieved with different sampling alternatives is observed. It is concluded, on the one hand, that a sampling scheme scarcely used in field archeology, sampling by interval transects, produces fewer errors. On the other hand, beyond the expected relation between the size of the sample and the precision with which a universe is represented, it has been possible to estimate which range of sampling fraction produces acceptable errors.

Key words: Subsurface evaluation, sampling schemes, sample fraction.

La discusión sobre los métodos y técnicas utilizadas en el trabajo de campo tuvo en las décadas de los años 70 y 80 del siglo pasado un importante papel en la consolidación de la arqueología como una ciencia a nivel global. El cuestionarse sobre la precisión y pertinencia de las distintas formas de obtener información básica sobre la cual se construye el razonamiento de los arqueólogos llevó tanto a mejorar la precisión del dato arqueológico, como a una mirada más crítica sobre los argumentos empíricos utilizados en la interpretación de los restos materiales depositados en los sitios arqueológicos. Uno de los aspectos de este debate metodológico desarrollado se centró en el problema del muestreo, bajo la premisa incluida por Thomas (1978) en su análisis de la horrible verdad sobre las estadísticas; todo trabajo arqueológico de campo se enfrenta con un problema de muestreo (Thomas 1978:232), ya sea que estemos consciente de su necesidad y recurramos a métodos como el muestreo probabilístico, o que pretendamos que nuestro criterio es el más adecuado al momento de seleccionar que parte de la realidad estudiaremos.

Dos de los campos metodológicos más desarrollados a partir de esta mirada se relacionaron

\footnotetext{
${ }^{1}$ Departamento de Antropología, Universidad Alberto Hurtado. Almirante Barroso 10, Santiago, Chile. lcornejo@uahurtado.cl
}

Recibido: septiembre 2018. Aceptado: enero 2019.

http://dx.doi.org/10.4067/S0717-73562019005001303. Publicado en línea: 12-junio-2019. 
precisamente con el papel del muestreo probabilístico durante la prospección (p.ej., Burger et al. 2004; Gallardo y Cornejo 1986; Muller 1974; Nance 1979; Plog 1976; Schiffer et al. 1978; Sundstrom 1993) y la evaluación de sitios por medio de recolecciones de superficie y sondeos subsuperficiales, ya sea con barrenos o pozos (p.ej., Chartkoff 1978; Cornejo et al. 1991; Cook y Burks 2011; Gallardo 1984; Hoffman 1993; Howell 1993; Kintigh 1986; Nance y Ball 1986; Shott 1985). En este último campo es donde precisamente pretendemos hacer un aporte aquí, centrándonos en evaluar conjuntamente dos aspectos esenciales al momento de seleccionar una determinada técnica de sondeo: el tamaño de la muestra y el esquema de muestreo probabilístico utilizado.

Nuestra evaluación del muestreo probabilístico aplicado a las técnicas de sondeo pretende poner énfasis en cómo diferentes combinaciones de dichas variables caracterizan la distribución de densidad de restos arqueológicos en los depósitos del sitio arqueológico, pero poniendo especial atención en dos aspectos poco considerados en los estudios citados previamente: la identificación de la forma del depósito arqueológico bajo la superficie y su tamaño. Estas dos dimensiones que forman parte de la definición de un sitio arqueológico son de especial importancia para definir, parafraseando a Gallardo (1984), dónde, cuánto y cómo excavar intensivamente el sitio, haciendo más certera la inversión de tiempo y recursos de este tipo de intervención ya sea en el campo de la investigación académica o en la toma de decisiones relativas al manejo del patrimonio arqueológico. A la vez, es cada vez más evidente que utilizar técnicas de menor costo, pero adecuadamente afinadas, tal como los pozos de sondeo o las recolecciones de superficie, permiten construir datos arqueológicos de escala regional adecuados para muchas de las preguntas que los y las arqueólogos(as) hoy habitualmente nos hacemos, sin la necesidad de recurrir a excavaciones intensivas (p.ej., Drennan y Peterson 2011; Falabella et al. 2014).

Este estudio se basa en la aplicación de distintos esquemas de muestreo y tamaños muestreales sobre sitios simulados con distintas distribuciones de densidades de materiales arqueológicos subsuperficiales $y$, a la vez, con diferentes tamaños de cada una de ellas. Cada aplicación de estos sondeos simulados es evaluada en términos de la precisión en que representan dichas variables.

\section{La Simulación}

La simulación de determinadas condiciones del registro arqueológico como una manera de evaluar las conclusiones posibles de extraer a partir de distintas técnicas analíticas ha sido una constante en el debate metodológico de la arqueología, ya sea en relación con la manera de cuantificar la alfarería (p.ej., Orton 1982) o de sus análisis químicos (p.ej., Neff et al. 1988), el desplazamiento de artefactos en campos cultivados (p.ej., Yorston et al. 1990), la diversidad del registro arqueológico (p.ej., McCartney y Glass 1990) o el actualmente popular análisis de suma de probabilidades de las dataciones de radiocarbono (Contreras y Meadows 2014).

Nuestra simulación se realizó a partir de sitios virtuales creados en un sistema de información geográfica ${ }^{1}$ sobre la base de un espacio de 200 x 250 metros donde se generó tres distintas distribuciones de densidades, las cuales se caracterizaron con un mapa de isocurvas de densidad para cada caso con una escala de cero a seis artefactos por litro. Estos tres sitios virtuales se caracterizan por la distinta complejidad en la forma en que estaban distribuidas las densidades de restos arqueológicos en los depósitos; un sitio Simple con una única concentración (Figura 1a), un Sitio Complejo 1 con dos concentraciones dentro del límite de un artefacto por litro (Figura 1b) y un sitio Complejo 2 con dos concentraciones separadas por un espacio con densidad cero (Figura 1c). En los tres casos se asumió que eventualmente la presencia de rasgos y materiales arqueológicos en superficie índica que, en toda el área virtual definida, un rectángulo de $200 \mathrm{~m}$ x $250 \mathrm{~m}$, podría haber materiales arqueológicos bajo la superficie, constituyéndose en el universo a muestrear. Esta definición basada en la información visible desde la superficie que correspondería a un plano de observación en los términos de la definición operacional del concepto de sitio arqueológico propuesta por Berenguer (1984:73), la cual en muchos casos, así como en las simulaciones aquí utilizadas, no es similar a la disposición subsuperficial de evidencias arqueológicas.

Sobre estos tres sitios virtuales se utilizó tres distintos esquemas de muestreo probabilístico habitualmente utilizados en este tipo de trabajo, cada uno con cuatro distintas frecuencias de muestreo. Los esquemas de muestreo utilizados fueron el sistemático alineado sobre la base de un cuadriculado rectangular (Figura 2a), en adelante SA, el sistemático no alineado sobre un cuadriculado rectangular (Figura 2b), en adelante $\mathrm{SN}$ y el sistemático alineado sobre una base radial (Figura 2c), también conocido como muestreo por transectos intervalares (Chartkoff 1978; Gallardo 1984), en adelante SR. A la vez, en cada sitio se utilizó cuatro frecuencias de muestreos definidas por la intensidad o separación entre los sondeos de 20 m, 30 m, 40 m y 50 $\mathrm{m}$, lo que implica una frecuencia de muestreo de $0,25 \%$, $0,11 \%, 0,06 \%$ y $0,04 \%$ respectivamente 2 .

En todos los casos se simuló que el sondeo empezó desde el centro del área virtual y se detuvo cuando se 


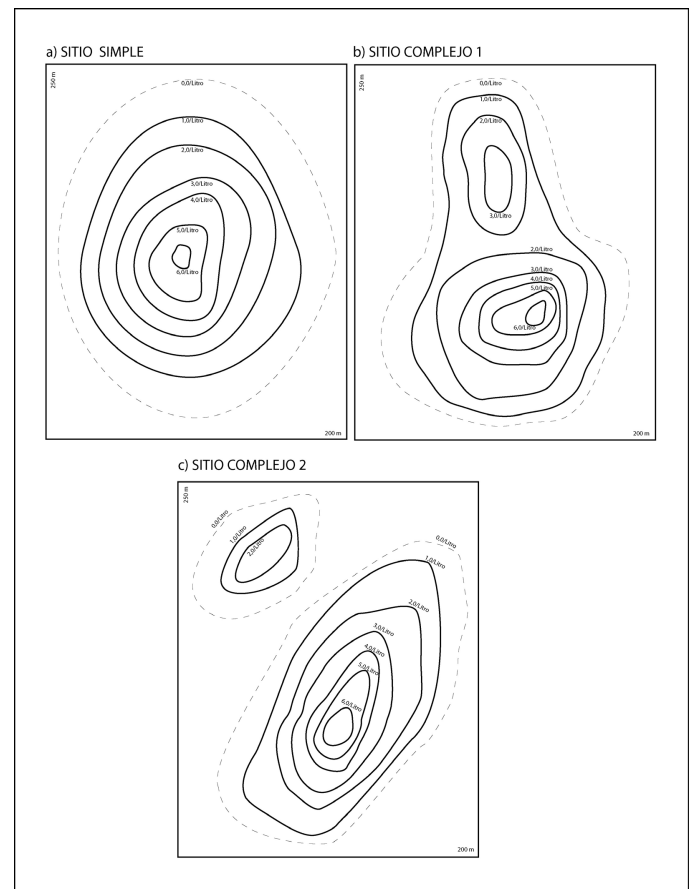

Figura 1. Distribución de las isocurvas de densidad en rangos de los restos arqueológico/litro de los tres sitios simulados.

Distribution in ranges of the density isocurves of the archaeological remains of the three simulated sites.

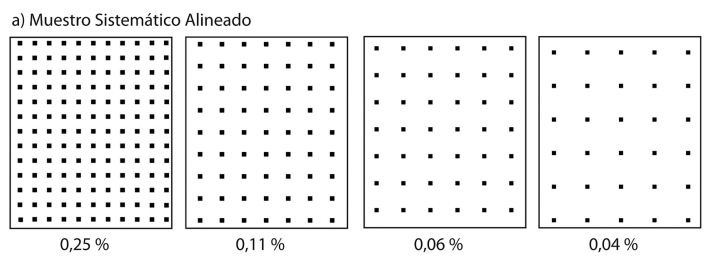

b) Muestro Sistemático No Alineado

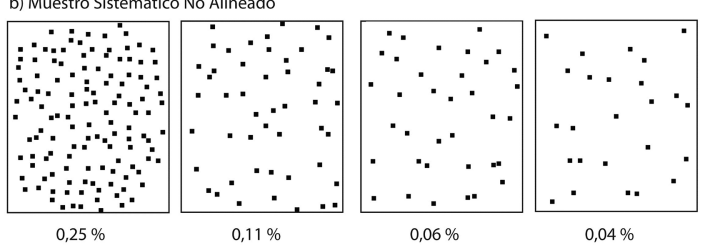

c) Muestro Sistemático Alineado Radial

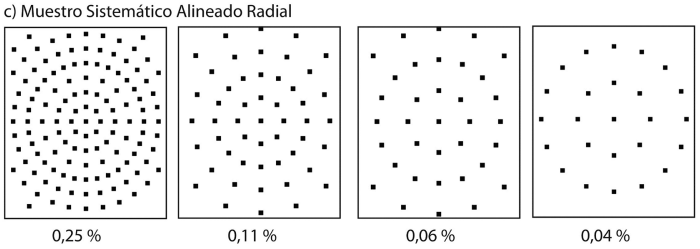

Nota: La representación de los pozos no esta a escala, pero siel tamaño del área donde se encontraría el sitio virtual.

Figura 2. Planteamiento de los sondeos simulados de acuerdo con los distintos esquemas de muestreo y fracción de la muestra.

Planting of the simulated soundings according to the different sampling and sample fraction schemes. 
encontraron al menos dos sondeos con densidad cero en el mismo eje, procedimiento que permite optimizar el número de sondeos utilizados. De esta manera, especialmente en los casos de las más altas frecuencias de muestreo se tuvo que "excavar" una cantidad distinta de sondeos para definir la periferia del sitio en términos subsuperficiales, ya que las distintas formas de la distribución de la densidad de restos subsuperficiales requerían cantidades variables de sondeos para definir su borde. En el caso de muestreo SR, ya que en algunos casos ocurre que en la medida que los transectos radiales se alejan del centro quedan espacios sin muestrear que son mayores que la intensidad definida por la fracción de la muestra, se introdujo transectos radiales cuyos sondeos no comienzan en el centro, sino que a la distancia suficiente para evitar este problema (p.ej., Figura 2c 0,25\%).

En esta simulación no se considera el tamaño del pozo de sondeo o el barrenado, ya que para los efectos prácticos de estimar la densidad de restos arqueológicos de un depósito esto es prácticamente irrelevante, ya que la densidad entendida como restos arqueológicos por unidad de volumen, generalmente 1 litro, anula las diferencias en el tamaño de la unidad de sondeo. No obstante, en los casos con densidades muy bajas el tamaño sí puede influir, ya que por ejemplo sondeos con barreno, cuyos diámetros mayores llegan a los $15 \mathrm{~cm}$, como señala McManamon (1984:259) sí bien pueden detectar suelos antrópicos o rasgos específicos, tienen menos probabilidad de rescatar restos arqueológicos, siendo posible obtener falsas densidades 0 (Howell 1993:482).

Es claro, no obstante, que al momento de ejecutar esta tarea en la realidad el tamaño de estas unidades de intervención sí es relevante e influye al menos en la visibilidad del excavador, la profundidad a la cual se puede excavar, la interpretación de la estratigrafía, la posibilidad de reconocer la diferencia entre rasgos y estratos o la capacidad para evadir posibles interferencias en el plano de excavación (p.ej., rocas).

Para cada sondeo virtual se calculó la densidad que se debiera tener dada la densidad conocida para cada punto dentro del sitio. Con esta información se creó una tabla de datos en el sistema de información geográfica y a partir de ella se construyó mapas de isocurvas de densidades ${ }^{3}$ producto de cada uno de los esquemas de muestreo probabilístico y fracción de la muestra seleccionados, definiendo áreas entre uno a seis artefactos por litro ${ }^{4}$. Los mapas de isocurvas obtenidos por cada tipo de muestreo probabilístico y por cada fracción de muestreo, fueron comparados con las isocurvas originales de los tres sitios virtuales considerando cuantas isocurvas fueron detectadas, la forma de las áreas definidas dentro de cada isocurva de densidad, su tamaño y su precisión espacial.
Respecto a la detección de las isocurvas se consignó la cantidad de isocurvas entre los valores un artefacto/litro y seis artefactos/litro, contabilizándose el número de isocurvas no generadas en cada caso. Para la estimación de la precisión de la forma se recurrió al índice morfométrico de compacidad $k_{c}$ (Zavoianu 1985) utilizado en geografía para definir la forma de las cuencas hidráulicas comparadas con la de un círculo que tiene la misma área, donde valores entre 1,00 y 1,25 representan una forma redonda a ovalada redonda, 1,25 a 1,50 representa una forma oval redonda a oval oblonga y entre 1,50 a 1,75 una forma de oval oblonga a rectangular oblonga. Así, dos áreas de isocurvas con valores $k_{c}$ similares debieran tener formas relativamente similares. Por otro lado, se calculó la superficie del espacio definido por cada isocurva virtual y se comparó con el área definida a partir de cada uno de los ensayos realizados. Por último, se midió la precisión espacial de cada isocurva calculada con la original, para lo cual se trazó sobre el centro de las concentraciones de cada uno de los sitios un sistema de eje cardinales que sirvió de guía para medir la distancia en metros entre la isocurva virtual original y la isocurva calculada a partir de cada uno de los esquemas y fracciones de la muestra utilizados sobre cada uno de los cuatro ejes cardinales (Figura 3).

Con el fin de establecer la precisión de cada esquema de muestreo probabilístico y de cada fracción de la muestra, así como la combinación de estas dos decisiones muestreales se calculó el error de las mediciones a partir de error relativo o incerteza fraccional (Félix y Greco 1968; Taylor 1997) definido por:

$$
e=\frac{\left(X_{m}-X_{r}\right)}{X_{m}}
$$

donde $X_{m}$ es el valor real y $X_{r}$ es el valor obtenido en cada ensayo.

En caso de la detección de las isocurvas originales se consideró como $X_{m}$ la cantidad de isocurvas reales y $X_{r}$ la cantidad de isocurvas detectadas por cada ensayo. Para la compacidad se utilizó el valor $k_{c}$ de cada área definida por las isocurvas originales como $X_{m}$ y el valor $k_{c}$ de las áreas de las isocurvas calculadas en cada ensayo como $X_{r}$. Para evaluar el tamaño la superficie del área dentro de cada isocurva se utilizó para $X_{m}$ el valor "real" de cada caso y como $X_{r}$ el valor calculado en cada muestreo. El caso de la precisión espacial es más complejo, ya que en principio el valor de $X_{m}$ debiera ser la coordenada de la intersección entre la isocurva y el eje cardinal utilizado para medir la distancia, valor cuya magnitud crece o disminuye dependiendo de la posición en que se encuentre la medida lo que afectaría artificialmente 


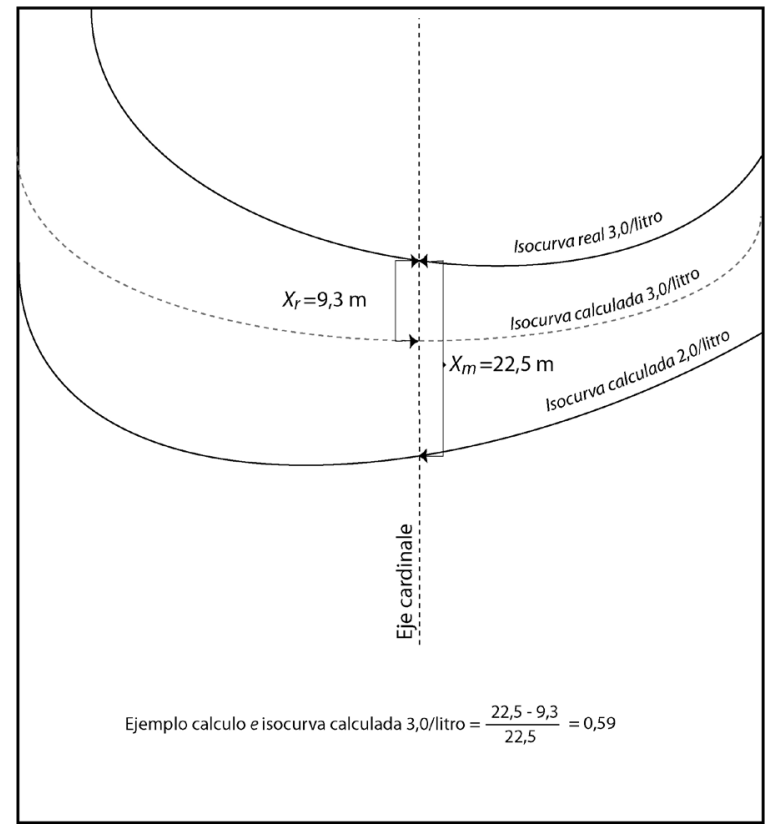

Figura 3. Método de cálculo de e (error relativo) para la precisión espacial de las isocurvas.

Method for calculating e (relative error) for the spatial accuracy of the isocurves.

la medición del error relativo . Por esta razón se asignó como $X_{m}$ el valor del espacio entre la isocurva real en cuestión con la isocurva real siguiente y el valor de $X_{r}$ se obtuvo midiendo la distancia entre las isocurvas real y calculada, lo que entrega una proporcionalidad que está ajustada a cada caso y no depende de su posición dentro del plano cartesiano (Figura 3).

Como regla de decisión para evaluar la significación del error relativo hay que considerar que el valor de $e$ $=0,0$ ocurre cuando no hay diferencia entre la medida original y la que se quiere evaluar, alcanzando el valor de 1,0 cuando la diferencia entre ambas es igual a la medida original y es mayor que 1,0 cuando el valor de la diferencia entre ambas medidas es superior al valor original. Así, asumimos que un valor de $e$ entre 0,0 y 0,25 representa un error relativo Bajo, entre 0,251 y 0,50 es Medio, entre 0,501 y 0,75 es Medio Alto, entre 0,751 y 1,0 es Alto y sobre 1,0 Muy Alto.

\section{Resultados}

Cada uno de los tres esquemas de muestreo probabilístico utilizados con cada una de las cuatro fracciones de la muestra seleccionadas produjo un conjunto de versiones distintas de la distribución de densidades de restos arqueológicos en los tres sitios virtuales que se diferencian en su complejidad, algunos ejemplos de los cuales se presentan en la Figura 4, siendo evidente que algunas combinaciones de estas tres variables descubren o ignoran una cantidad distinta de isocurvas de densidad de restos arqueológicos de cada uno de los tipos de sitios virtuales generando distintos grados de error. La curva menos detectada fue la de 6 artefactos/litro, la cual realmente fue reconocida en solo 5 de los 36 ensayos realizados y un $e=0,86$ (Figura 5). Esta situación está claramente relacionada con el diámetro máximo del área incluida dentro de esta isocurva en los sitios virtuales (Sitio Simple $=16,1 \mathrm{~m}$, Sitio Complejo 1=18,7 m y Sitio Complejo 2=27,3 m), dos de los cuales son menores que la intensidad mayor de muestreo de $20 \mathrm{~m}$ aquí utilizada. De hecho, en el caso del sitio Complejo 2, donde el diámetro mayor del área de la isocurva de 6 artefactos/litro es levemente superior que la intensidad mayor de muestreo, esta curva sí fue relevada en tres de los ejercicios realizados.

La falta de detección de isocurvas nos señala que mientras el muestreo SR tiene todos sus ejercicios en el rango de error Bajo (Tabla 1), con un par de casos de $e=0$, en el muestreo SN esta proporción disminuye a la mitad y una fracción importante de ejercicios realizados con este muestreo se encuentran en el rango de error Medio (25,0\%). Por su parte, el muestreo SA se encontraría en una posición intermedia, aunque se debe señalar que una parte significativa de sus resultados están en el rango de error Bajo. Al considerar cómo influyó en los resultados la fracción o tamaño de la muestra se aprecia que, tal como sería esperable, hay una tendencia a que la disminución en el tamaño de 


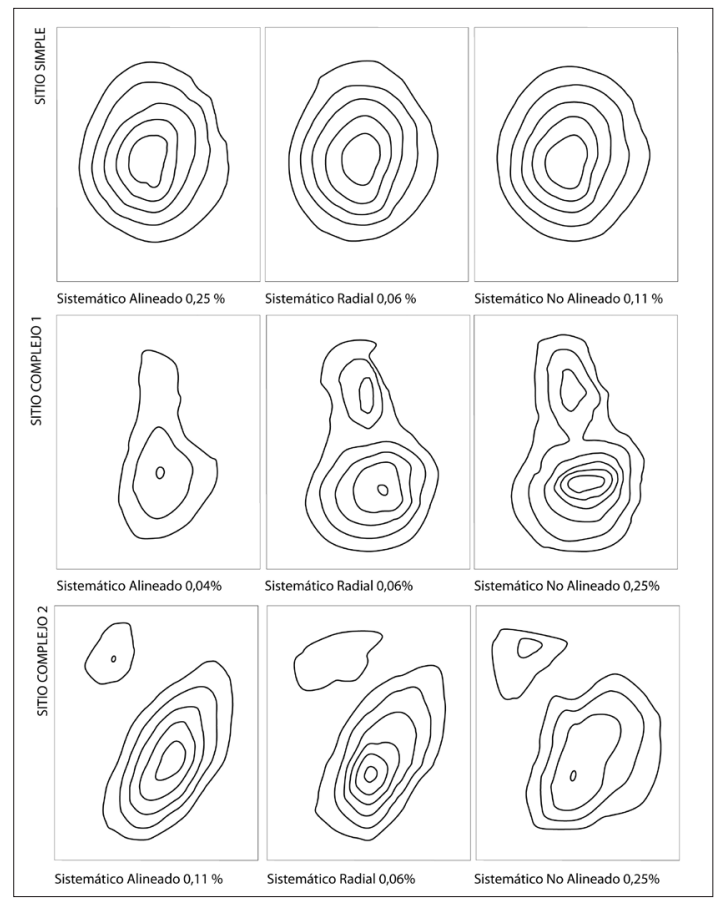

Figura 4. Ejemplos de distribuciones de las isocurvas obtenidas con distintos esquemas de muestreo y tamaño de la muestra.

Examples of distributions of the isocurves obtained with different sampling and sample size schemes.

la muestra está acompañada de una disminución en la cantidad de casos que se encuentran más allá de la región de error Bajo (Tabla 2), aunque la correlación de ambas variables resulta baja $\left(r^{2}=0,21\right)$. Este último resultado, sin embargo, debiera ser un artefacto de las magnitudes pequeñas en cuestión, ya que basta con un par de casos para alterar el valor de esta correlación.

Por último, si consideramos el grado de complejidad de los sitios podemos ver que tal como era esperable el Sitio Simple fue el con menor error, ya que en todos los ensayos realizados faltó solo una isocurva, con un valor en todos de $e=0,17$ es decir todos en el rango de error Bajo (Tabla 3). En el caso de los sitios complejos la expectativa era que el muestreo en el Sitio Complejo 2 tuviera más errores debido a que consideramos que la separación de dos concentraciones por un espacio de 0 artefactos/litro, con un ancho mínimo de solo $13 \mathrm{~m}$, fuera una dificultad dado las intensidades resultantes de las fracciones de muestreo (p.ej., Way 2017). Sin embargo, desde el punto de vista de la cantidad de isocurvas detectadas fue en el Sitio Complejo 1 donde se produjeron los mayores errores, con un $33,3 \%$ en el rango de error medio. Por su parte el sitio Complejo 2, se ubicó en una posición intermedia, aunque no presenta ningún ejercicio en un rango superior a Medio.

Al considerar la precisión en la forma de las áreas de cada isocurva por medio del índice de compacidad $\left(k_{c}\right)$ se observa que en términos generales todas las isocurvas "reales" de los sitios virtuales producen áreas

Tabla 1. Número y frecuencia porcentual de ensayos de los esquemas de muestreo en los distintos rangos de error del número de isocurvas detectadas.

Number and percentage frequency of sample scheme trials in the different error ranges of the number of isocurves detected.

\begin{tabular}{cccc}
\hline & Muestreo SA & Muestreo SN & Muestreo SR \\
\hline Bajo & $10(83,3)$ & $9(75,0)$ & $12(100,0)$ \\
Medio & $2(16,7)$ & $3(25,0)$ & $0(0,0)$ \\
Medio Alto & $0(0,0)$ & $0(0,0)$ & $0(0,0)$ \\
Alto & $0(0,0)$ & $0(0,0)$ & $0(0,0)$ \\
Muy Alto & $0(0,0)$ & $0(0,0)$ & $0(0,0)$ \\
\hline
\end{tabular}

Tabla 2. Número y frecuencia porcentual de ensayos de las fracciones de muestreo en los distintos rangos de error del número de isocurvas detectadas.

Number and percentage frequency of trials of the sampling fractions in the different error ranges of the number of isocurves detected.

\begin{tabular}{ccccc}
\hline & $0,25 \%$ & $0,11 \%$ & $0,06 \%$ & $0,04 \%$ \\
\hline Bajo & $7(77,8)$ & $8(88,9)$ & $6(66,7)$ & $6(66,7)$ \\
Medio & $2(22,2)$ & $1(11,1)$ & $3(33,3)$ & $1(1,1)$ \\
Medio Alto & $0(0)$ & $0(0)$ & $0(0)$ & $2(22,2)$ \\
Alto & $0(0)$ & $0(0)$ & $0(0)$ & $0(0)$ \\
Muy Alto & $0(0)$ & $0(0)$ & $0(0)$ & $0(0)$ \\
\hline
\end{tabular}




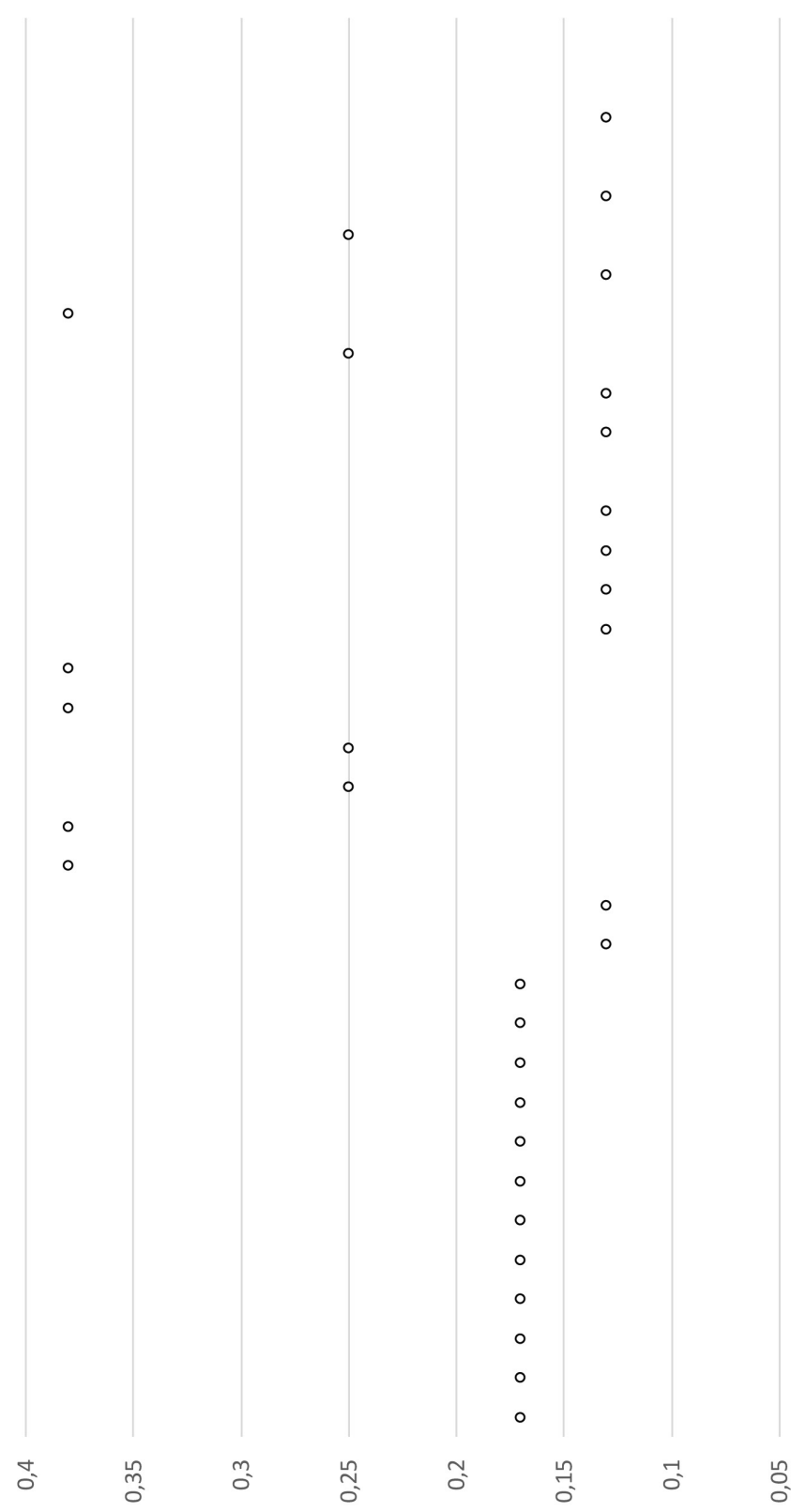

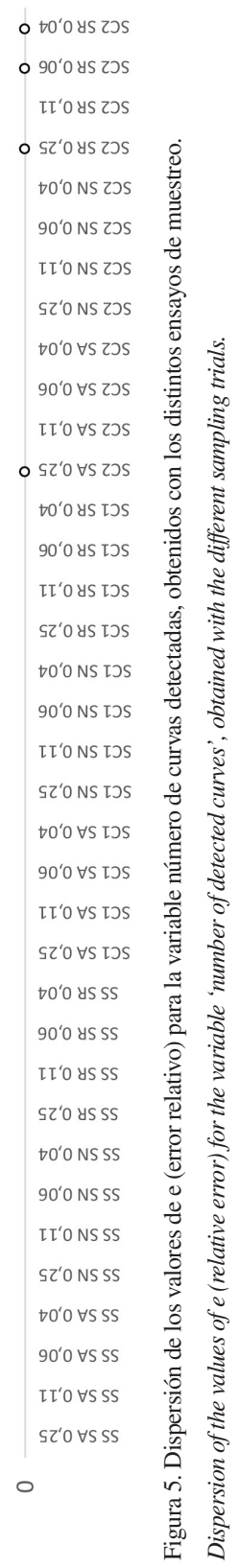

(ә) ом!̣ерәд лоднә 
Tabla 3. Número y frecuencia porcentual de ensayos de los sitios virtuales en los distintos rangos de error del número de isocurvas detectadas.

Number and percentage frequency of trials of the virtual sites in the different error ranges for the number of isocurves detected.

\begin{tabular}{cccc}
\hline & Sitio Simple & Sitio Complejo 1 & Sitio Complejo 2 \\
\hline Bajo & $12(100,0)$ & $8(66,7)$ & $11(91,7)$ \\
Medio & $0(0,0)$ & $4(33,3)$ & $1(8,3)$ \\
Medio Alto & $0(0,0)$ & $0(0,0)$ & $0(0,0)$ \\
Alto & $0(0,0)$ & $0(0,0)$ & $0(0,0)$ \\
Muy Alto & $0(0,0)$ & $0(0,0)$ & $0(0,0)$ \\
\hline
\end{tabular}

que se encuentran entre valores de 1,00 y $1,25 k$ por lo cual pueden ser descritas como tendientes a redondas y ovaladas redondas. Esta descripción de hecho prácticamente no varía con los distintos esquemas de muestreo, las fracciones de la muestra utilizadas o la complejidad de los sitios, resultando casi todos los ensayos en la región de error Bajo (Figura 6). Son excepcionales dos ejercicios realizados en el muestreo SA y tres en el muestreo SN en el sitio Complejo 1 con valores de $k_{c}$ que señalarían que las áreas bajo las isocurvas en cuestión irían de ovaladas redondas a ovaladas oblongas, aunque sólo dos de ellos realizados con el esquema de Muestreo SN estarían en la región de error Medio (Figura 6). Cabe señalar que ni en este análisis ni en los siguientes incluiremos los casos en que no se detectó una determinada isocurva, lo que ya se consideró previamente al momento de evaluar la complejidad del sitio, ya que no es posible comparar un valor conocido contra uno inexistente.

El tamaño de áreas dentro de las isocurvas proyectados por los distintos ensayos entrega resultados un poco distintos, ya que ahora sí se aprecian errores mayores que se concentran en determinados ejercicios (Figura 7). Los resultados de los distintos tipos de muestreos (Tabla 4) muestran que mientras el muestreo SA y SR tienen más del $80 \%$ de sus ejercicios en el rango de error Bajo, con valores medio $e$ de $0,145(s=0,195)$ y $0,144(s=0,181)$ respectivamente, el muestreo $\mathrm{SN}$ solo tiene cerca de un $60 \%$ en dicho rango de error y alcanzando una $\bar{x} e=0,362(s=0,480)$. De hecho, en este último esquema es donde se encuentran todos los valores de $e$ superiores a 1,0, ubicándose en la zona de error Muy Alto más del 10\% de los ejercicios. Desde el punto de vista de la fracción de la muestra es posible apreciar (Tabla 5) que, tal como era esperable, se produce un aumento en la media del error relativo con la disminución del tamaño de la muestra, así la fracción $0,25 \%$ tiene un $\bar{x} e=0,18(s=0,36)$, la fracción $0,11 \%$ tiene un $\bar{x} e=0,18(s=0,35)$, la fracción $0,06 \%$ tiene un $\bar{x} e=0,26(s=0,38)$ y la fracción $0,04 \%$ tiene un $\bar{x} e=0,24(s=0,27)$, existiendo entre ambas variables una correlación inversa $\left(r^{2}=0,60\right)$. Este efecto se hace más claro cuando se observa la cantidad de ensayos con cada fracción de la muestra que se encuentran en el rango de Bajo error relativo (Tabla 5), con una alta correlación positiva entre la disminución en cantidad de ensayos y la disminución en los tamaños de la muestra $\left(r^{2}=0,88\right)$. Por último, si consideramos la complejidad de los sitios virtuales que se definió para este estudio, se repite la situación vista previamente, donde el Sitio Complejo 1 resulta ser el que produce muestreos con mayores errores relativos ( $\overline{\boldsymbol{x}} e=0,37 s=0,47)$, mientras que el sitio Simple es por lejos el que tiene menores errores relativos ( $\bar{x} e=0,07 s=0,11)$. El sitio Complejo 2. por su parte, se encuentra en una posición intermedia $(\bar{x} e=0,18 s=0,20)$. De hecho, cuando miramos la proporción de ensayos que se encuentran en los distintos rangos de error relativo (Tabla 6), se constata que casi el 20\% de los ensayos realizados en el sitio Complejo 1 se encuentran en los rangos de error relativo Alto y Muy Alto.

Tabla 4. Número y frecuencia porcentual de ensayos de los esquemas de muestreo en los distintos rangos de error de tamaño de las isocurvas.

Number and percentage frequency of trials of the sampling schemes in the different error ranges of the size of the isocurves.

\begin{tabular}{cccc} 
& Muestreo SA & Muestreo SN & Muestreo SR \\
\hline Bajo & $56(81,2 \%)$ & $40(59,7 \%)$ & $63(81,8 \%)$ \\
Medio & $9(13,0 \%)$ & $8(11,9 \%)$ & $10(13,0 \%)$ \\
Medio Alto & $2(2,9 \%)$ & $7(10,4 \%)$ & $3(3,9 \%)$ \\
Alto & $2(2,9 \%)$ & $5(7,5 \%)$ & $1(1,3 \%)$ \\
Muy Alto & $0(0 \%)$ & $7(10,5 \%)$ & $0(0 \%)$
\end{tabular}

Tabla 5. Número y frecuencia porcentual de ensayos de las fracciones de muestreo en los distintos rangos de error del tamaño de las isocurvas.

Number and percentage frequency of trials of the sampling fractions in the different error ranges of the size of the isocurves.

\begin{tabular}{ccccc}
\hline & $0,25 \%$ & $0,11 \%$ & $0,06 \%$ & $0,04 \%$ \\
\hline Bajo & $46(79,3 \%)$ & $42(77,8 \%)$ & $37(71,2 \%)$ & $34(69,4 \%)$ \\
Medio & $7(12,1 \%)$ & $7(13,0 \%)$ & $6(11,5 \%)$ & $7(14,3 \%)$ \\
Medio Alto & $2(3,4 \%)$ & $1(1,9 \%)$ & $5(9,6 \%)$ & $4(8,2 \%)$ \\
Alto & $0(0,0 \%)$ & $3(5,6 \%)$ & $1(1,9 \%)$ & $4(8,2 \%)$ \\
Muy Alto & $3(5,2 \%)$ & $1(1,9 \%)$ & $3(5,8 \%)$ & $0(0,0 \%)$ \\
\hline
\end{tabular}

Tabla 6. Número y frecuencia porcentual de ensayos de los sitios virtuales en los distintos rangos de error del tamaño de las isocurvas.

Number and percentage frequency of trials of the virtual sites in the different error ranges of the size of the isocurves.

\begin{tabular}{cccc}
\hline & Sitio Simple & Sitio Complejo 1 & Sitio Complejo 2 \\
\hline Bajo & $55(91,7 \%)$ & $46(63,0 \%)$ & $58(72,5 \%)$ \\
Medio & $5(8,3 \%)$ & $8(11,0 \%)$ & $14(17,5 \%)$ \\
Medio Alto & $0(0,0 \%)$ & $5(6,8 \%)$ & $7(8,8 \%)$ \\
Alto & $0(0,0 \%)$ & $7(9,6 \%)$ & $1(1,3 \%)$ \\
Muy Alto & $0(0,0 \%)$ & $7(9,6 \%)$ & $0(0,0 \%)$ \\
\hline
\end{tabular}




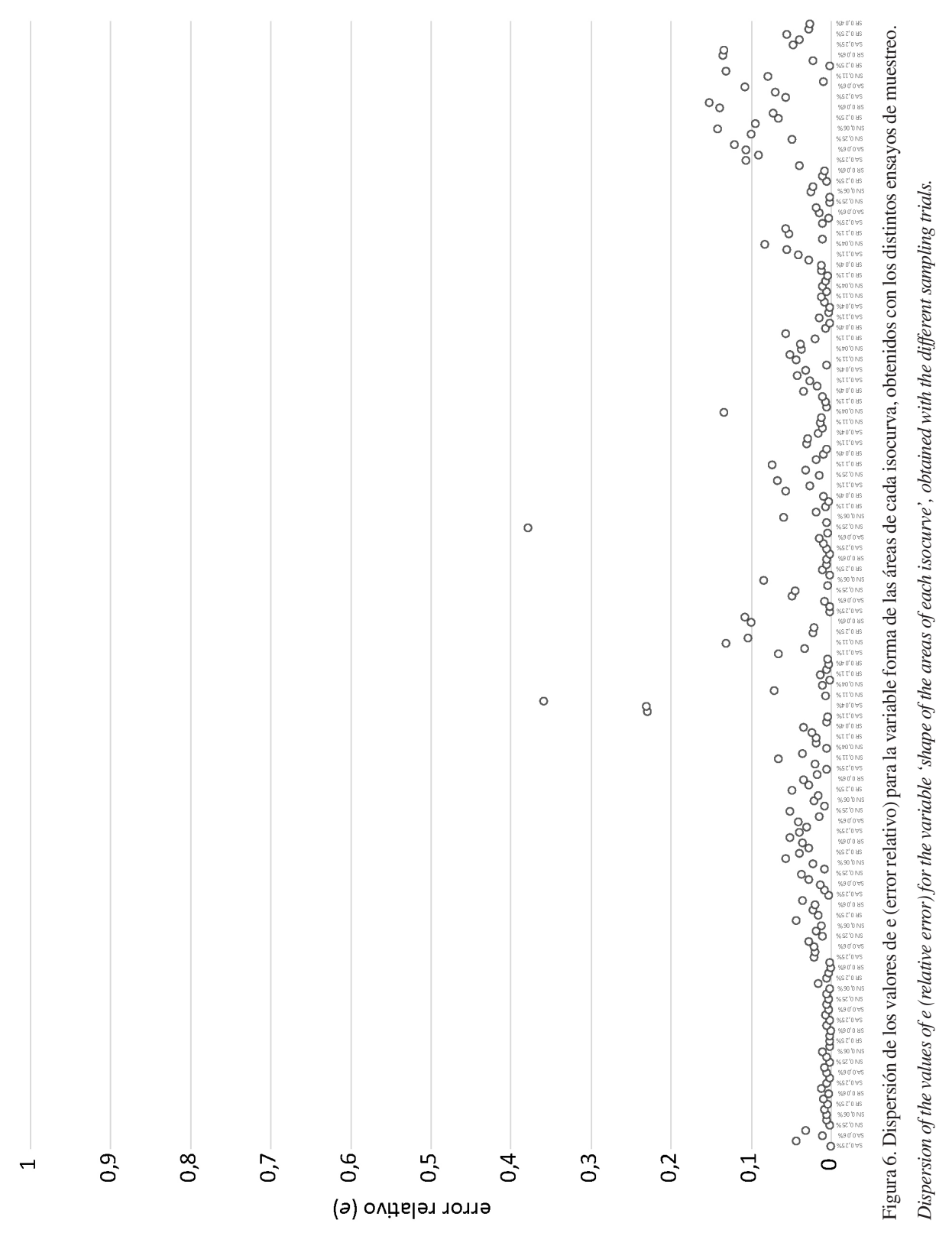


436

Luis E. Cornejo B.

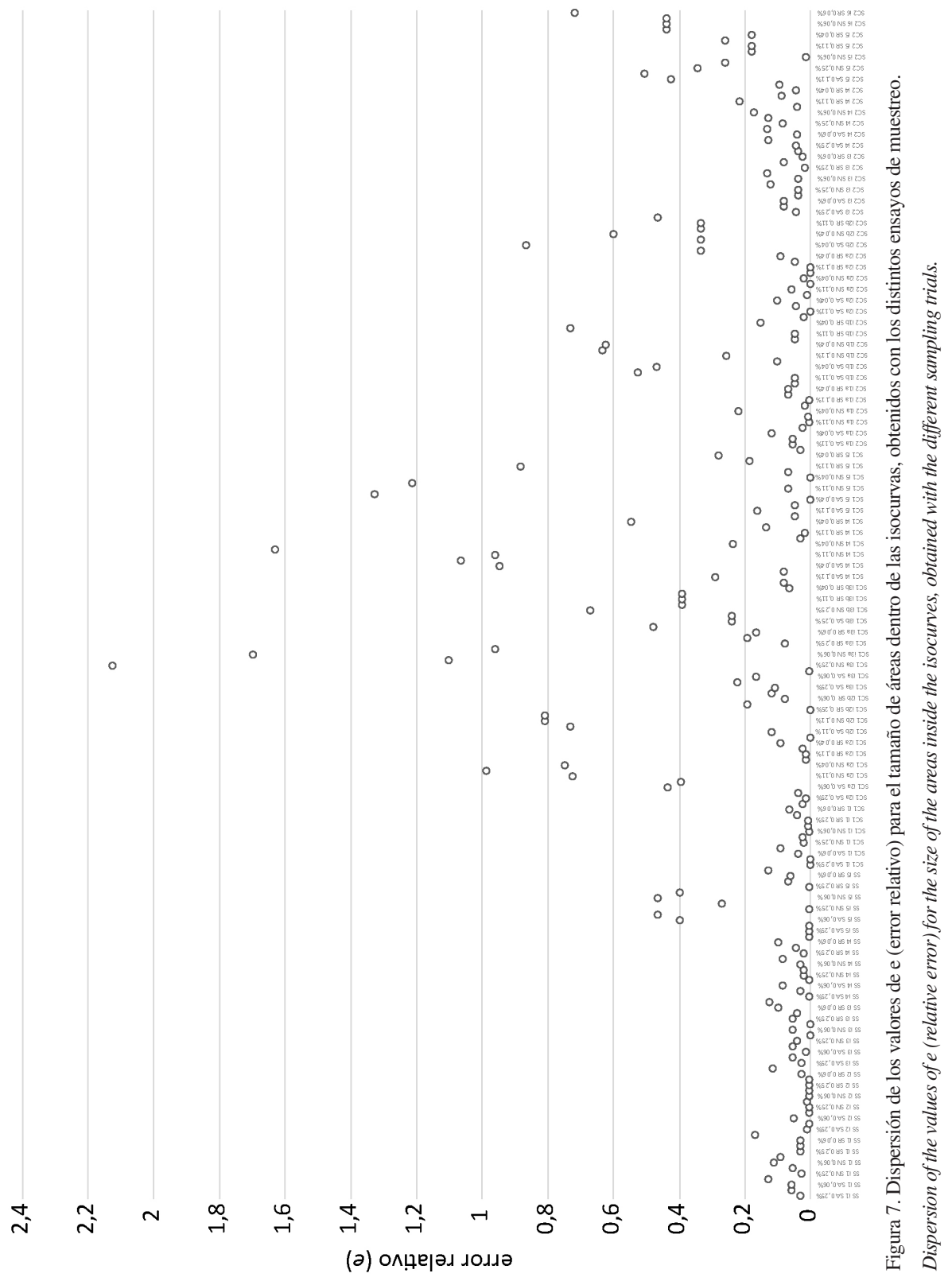


Nuestra tercera y última variable de análisis es la precisión con que se dibujan sobre el terreno virtual las isocurvas dependiendo del esquema de muestreo probabilístico y fracción de la muestra utilizados. En este caso los resultados difieren de los anteriores ya que ahora se observan una mayor cantidad de ensayos que presentan errores altos, muchos de los cuales se encuentran en la categoría de error Alto e incluso alcanzando algunos valores muy por sobre los vistos previamente (Figura 8).

8

7

$\circ \quad \circ \quad \circ$

6

5

$\frac{0}{0}$
0
$\frac{1}{0}$
$\frac{0}{0}$
$\frac{1}{2}$
$\frac{2}{0}$

4

$\frac{1}{2}$

3

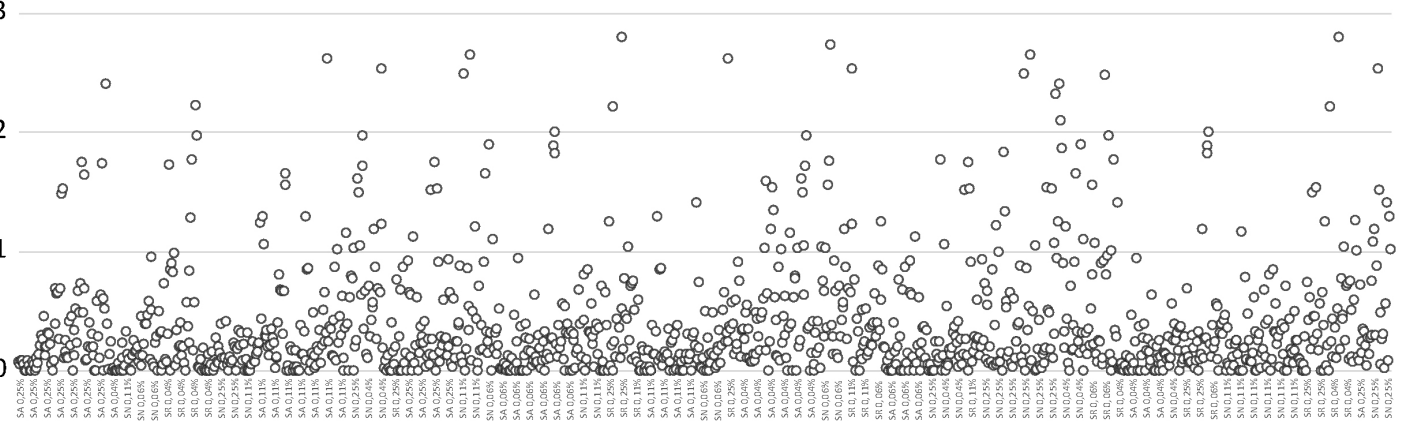

Figura 8. Dispersión de los valores de e (error relativo) para la precisión con que se dibujan sobre el terreno virtual las isocurvas, obtenidos con los distintos ensayos de muestreo.

Dispersion of the values of e (relative error) for the accuracy with which the isocurves are displayed on the virtual land, obtained with the different sampling trials.

Desde el punto de vista de los esquemas de muestreo probabilístico esta vez es evidente que el muestreo SR tiene la mayor proporción de sus ensayos en el rango de error Bajo y con la menor proporción en el rango de error Muy Alto (Tabla 7), lo aue se ve reflejado claramente en la media más baja $(\bar{x} e=0,33)$ y la menor dispersión en torno a ella reflejada en la desviación estándar $(s=0,45)$. En el lado opuesto el muestreo SN, si bien presenta la segunda proporción de casos en el rango de error Bajo, también presenta la mayor proporción de ensayos en el rango de error Alto (Tabla 7) y con la media más alta $(\bar{x} e=0,45)$ con una alta dispersión en torno a ella $(s=0,65)$. El muestreo SA, por su parte, si bien alcanza una proporción intermedia de ensayos en el rango de error Alto, también presenta la menor proporción en el rango de error Bajo, lo que produce que su valor medio y de dispersión sean muy parecidos al muestreo $\mathrm{SN}(\bar{x} e=0,45 s=0,60)$.
Considerando el efecto de la fracción de la muestra los resultados, como ya se dijo, se encuentran dentro de lo esperable. En la medida que la muestra se hace

Tabla 7. Número y frecuencia porcentual de ensayos de los esquemas de muestreo en los distintos rangos de error de la precisión espacial de las isocurvas.

Number and percentage frequency of the trials of the sampling schemes in the different error ranges of the spatial accuracy of the isocurves.

\begin{tabular}{cccc}
\hline & Muestreo SA & Muestreo SN & Muestreo SR \\
\hline Bajo & $150(50,0 \%)$ & $160(55,4 \%)$ & $187(57,7 \%)$ \\
Medio & $70(23,3 \%)$ & $46(15,9 \%)$ & $79(24,4 \%)$ \\
Medio Alto & $35(11,7 \%)$ & $23(8 \%)$ & $24(7,4 \%)$ \\
Alto & $13(4,3 \%)$ & $23(8 \%)$ & $8(2,5 \%)$ \\
Muy Alto & $32(10,7 \%)$ & $37(12,8 \%)$ & $26(8 \%)$ \\
\hline
\end{tabular}


más pequeña disminuye proporcionalmente la cantidad de ensayos en el rango de error Bajo (Tabla 8) con una correlación directa muy marcada $\left(r^{2}=0,99\right)$. A la vez se produce prácticamente la misma intensidad de correlación $\left(r^{2}=0,93\right)$, aunque inversamente proporcional, entre la disminución del tamaño de la muestra y el aumento en el número de ensayos en el rango de error Muy Alto. Por último, considerando la complejidad de los sitios virtuales nuevamente, como era esperable, el Sitio Simple es el que tiene la mayor proporción de sus ensayos en el rango de error Bajo y la menor en el rango de error Muy Alto (Tabla 9). En este caso, sin embargo, los sitios Complejos 1 y 2 según sus proporciones en los rangos de error Bajo y Muy Alto, resultan ser prácticamente iguales. Esto se ve reforzado por los valores medios de error relativo obtenidos para cada tipo de sitio (Sitio Simple $\bar{x} e=0,31 s=0,42$, Sitio Complejo $1 \bar{x} e=0,44 s=0,71$ y Sitio Complejo $2(\bar{x}$ $e=0,42 s=0,50$ ), separándose claramente el Sitio Simple de los otro.

Tabla 8. Número y frecuencia porcentual de ensayos de las fracciones de muestreo en los distintos rangos de error de la precisión espacial de las isocurvas.

Number and percentage frequency of the trials of the sampling fractions in the different error ranges of the spatial accuracy of the isocurves.

\begin{tabular}{ccccc}
\hline & $0,25 \%$ & $0,11 \%$ & $0,06 \%$ & $0,04 \%$ \\
\hline Bajo & $195(78,6 \%)$ & $121(53,1 \%)$ & $99(44,4 \%)$ & $82(38,3 \%)$ \\
Medio & $33(13,3 \%)$ & $57(25 \%)$ & $56(25,1 \%)$ & $49(22,9 \%)$ \\
Medio Alto & $9(3,6 \%)$ & $23(10,1 \%)$ & $20(9,0 \%)$ & $30(14,0 \%)$ \\
Alto & $6(2,4 \%)$ & $8(3,5 \%)$ & $15(6,7 \%)$ & $15(7,0 \%)$ \\
Muy Alto & $5(2,0 \%)$ & $19(8,3 \%)$ & $33(14,8 \%)$ & $38(17,8 \%)$ \\
\hline
\end{tabular}

Tabla 9. Número y frecuencia porcentual de ensayos de los sitios virtuales en los distintos rangos de error de la precisión espacial de las isocurvas.

Number and percentage frequency of the trials of the virtual sites in the different error ranges of the spatial accuracy of the isocurves.

\begin{tabular}{cccc}
\hline & Sitio Simple & Sitio Complejo 1 & Sitio Complejo 2 \\
\hline Bajo & $152(63,3 \%)$ & $177(51,9 \%)$ & $168(50,6 \%)$ \\
Medio & $44(18,3 \%)$ & $73(21,4 \%)$ & $78(23,5 \%)$ \\
Medio Alto & $21(8,8 \%)$ & $36(10,6 \%)$ & $25(7,5 \%)$ \\
Alto & $7(2,9 \%)$ & $21(6,2 \%)$ & $16(4,8 \%)$ \\
Muy Alto & $16(6,7 \%)$ & $34(10,0 \%)$ & $45(13,6 \%)$ \\
\hline
\end{tabular}

\section{Conclusiones}

Los resultados previamente descritos pueden ser integrados de manera que sea posible concluir cuales son las condiciones (esquema de muestreo probabilístico ejecutado, la fracción de la muestra utilizada y la complejidad de los sitios virtuales muestreados) que inducen una mejor representación de los sitios virtuales. Para esto hemos recurrido a la media de las frecuencias porcentuales que en cada caso se obtuvieron para los rangos de error relativo (Bajo, Medio, Medio Alto, Alto y Muy Alto) para las variables tamaño de las áreas, número de isocurvas y precisión espacial (Tabla 10). Tomando de estos sólo los valores de los rangos de error Bajo y Muy Alto, es decir los que polarizan los resultados, podemos analizar de una manera más sintética nuestros ensayos. Queda claro de esta manera que hay determinadas decisiones de muestreo para la aplicación de sondeos que producen mejores imágenes de la densidad de restos en los depósitos de los sitios arqueológicos que otras. A la vez es evidente que estos resultados están en directa relación con la complejidad de los sitios, aspecto que obviamente no es posible conocer antes de realizar una tarea de caracterización del tipo aquí estudiada.

Desde el punto de vista de las decisiones tomadas por el/la arqueólogo/a, muy en la línea de lo esperado según la teoría básica del muestreo probabilístico, a un mayor tamaño de la muestra se obtienen mejores resultados. Las fracciones de 0,25\% y $0,11 \%$, que en el marco de espacio virtual creado implican una intensidad de 20 y 30 metros, son las que ofrecen una buena representación de los sitios respectivamente, con una media de $78,6 \%$ y un 73,3 $\%$ en el rango de error Bajo y un 2,4 \% y 3,4\% en el rango de error Muy Alto. Muestras de menor tamaño, consecuentemente, resultarían insatisfactorias (Tabla 10). Tomando comparativamente los valores medios de las frecuencias porcentuales que tienen en los rangos de error muestral Bajo la diferencia porcentual entre la muestra de las fracciones de mayor tamaño y menor tamaño $(0,25 \%$ y $0,04 \%)$ resulta ser de un $26,0 \%$ y de $146,1 \%$ si consideramos el rango de error Muy Alto. Por su parte la diferencia entre las muestras $0,25 \%$ y $0,11 \%$ son sólo de 6,8 \% en el rango de Bajo error y de 41,8\% en el rango de error Muy Alto. A su vez, la fracción de la muestra de $0,06 \%$ se encuentra a una distancia porcentual de la mayor $(0,25 \%)$ de $22,7 \%$ en el rango error Bajo y de 185,4 \% en el rango Muy Alto, es decir muy similar a la fracción de muestreo menor $(0,04 \%)$. De hecho, si ubicamos estos valores en un plano cartesiano (Figura 9), resulta que mientras las fracciones de $0,25 \%$ y $0,11 \%$ sólo se encuentran a una distancia euclidiana de 5,4, la fracción de $0,25 \%$ se aleja un 20,7 de la fracción $0,04 \%$ y un 18,3 de la fracción $0,06 \%$.

Enfocándonos en el esquema de muestreo probabilístico utilizado en estos ensayos, resulta interesante observar que el Muestreo Sistemático Radial (SR), también conocido como muestreo por transectos intervalares (Chartkoff 1978) y cuyo uso no es tan extensivo, resulta ser el que brinda mejores resultados (Tabla 10), con una media del 79,8\% de los ensayos en el rango de error Bajo y solo el 2,7 \% en 
Tabla 10. Valor medio de la frecuencia porcentual en los rangos de error relativo de las distintas variables en que se aplicaron los muestreos.

Medium value of the percentage frequency in the relative error ranges of the different variables on which the samplings were applied.

\begin{tabular}{cccccc}
\hline & Bajo & Medio & Medio Alto & Alto & Muy Alto \\
\hline Sitio Simple & 85,0 & 8,9 & 2,9 & 1,0 & 2,2 \\
Sitio Complejo 1 & 55,0 & 21,9 & 11,4 & 5,3 & 6,5 \\
Sitio Complejo 2 & 66,0 & 22,0 & 5,4 & 2,0 & 4,5 \\
Fracción 0,25\% & 78,6 & 15,9 & 2,3 & 0,8 & 2,4 \\
Fracción 0,11\% & 73,3 & 16,4 & 4,0 & 3,0 & 3,4 \\
Fracción 0,06\% & 60,8 & 23,3 & 6,2 & 2,9 & 6,9 \\
Fracción 0,04\% & 58,1 & 12,8 & 14,8 & 5,1 & 5,9 \\
Muestreo SA & 68,7 & 17,7 & 7,6 & 2,4 & 3,6 \\
Muestreo SN & 55,0 & 23,2 & 8,9 & 5,1 & 7,8 \\
Muestreo SR & 79,8 & 12,5 & 3,8 & 1,3 & 2,7 \\
Número de Curvas & 81,7 & 14,8 & 3,5 & 0 & 0 \\
Tamaño & 74,8 & 12,6 & 5,5 & 3,8 & 3,3 \\
Precision Espacial & 54,3 & 21,3 & 9,1 & 4,8 & 10,5 \\
\hline
\end{tabular}

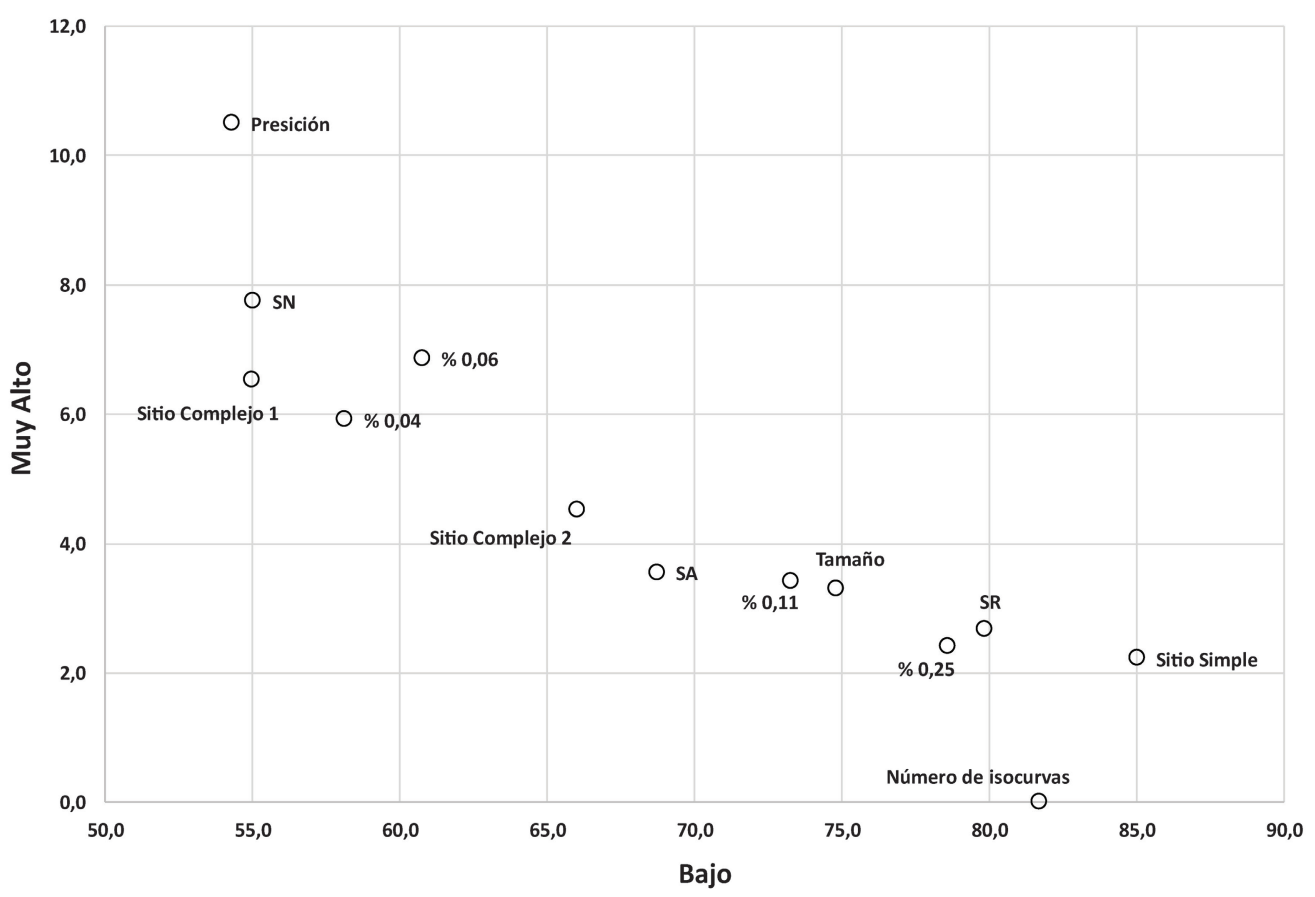

Figura 9. Dispersión en el plano cartesiano de la media de las frecuencias porcentuales para los rangos de error Muy Alto y Bajo de los distintos esquemas de muestreo, tamaños de la muestra y tipos de sitios virtuales.

Dispersion on the Cartesian plane of the median of the percentage frequencies for the error ranges Very High and Low for the different sampling schemes, sample sizes and types of virtual sites. 
el rango de error Muy Alto. Por su parte el Muestreo Sistemático No Alineado (SN) demuestra ser el menos preciso ya que solo una media de $55,0 \%$ de los ensayos se encuentran en el rango de error Bajo y un 7,8\% en el rango Muy Alto. Esta diferencia representa un 31,1 $\%$ en el rango de error Bajo y 189,7 \% en el rango de error Muy Alto. Por su parte el muestreo Sistemático Alineado (SA), si bien ocuparía el segundo lugar en su precisión para representar los sitios, solo tiene una diferencia con SR de 13,9\% en el rango Bajo y de 32,9\% en rango Muy Alto. Al trasladar estos valores al plano cartesiano (Figura 9) resulta que mientras la distancia entre el muestreo SR y el SN es de 23,3\%, la distancia entre los muestreos SR y SA.

Considerando las características del sitio, en todos los ensayos que hemos realizados es el Sitio Simple, es decir que tiene una sola concentración, el que puede ser representado con menos error, con una media del $85,0 \%$ de los ensayos en el rango de error Bajo y un 2,2\% en el rango de error Muy Alto. Por el contrario, el Sitio Complejo 1, que tiene dos concentraciones dentro de la isocurva de 1 artefacto/litro, resulta representado con mayor error, llegando solo una media del 55,0\% de los ensayos en el rango de error Bajo y $6,5 \%$ en el rango Muy Alto. Al comparar las frecuencias porcentuales en el rango de error Bajo de estos dos sitios se observa que existe una diferencia porcentual en la calidad en que se representan los sitios de un 35,3\%. En el otro extremo, cuando se realiza la misma relación en el rango de error Muy Alto la diferencia es de un 193,6\%. Esta distancia disminuye si comparamos el Sitio Simple con el Sitio Complejo 2, alcanzando los valores de 22,3\% y 103,3\% en los rangos Bajo y Muy Alto. Ubicando los tres sitios en el plano cartesiano con estos rangos de error (Figura 9), vemos que la distancia euclidiana entre el Sitio Simple y el Sitio Complejo 1 en de 30,3 mientras que la distancia del Sitio Simple con el sitio Complejo 2 es de solo 19,1.

Por último, también podemos aquí considerar cómo las decisiones de muestreo afectaron a las variables que utilizamos precisamente para evaluar la eficiencia de ellos, considerando también la media de error medio que cada una de ellas produjo en los distintos ensayos (Tabla 10). En primer lugar, es evidente que el Número de Curvas detectados es el que menos error presentó, con una frecuencia media de $81,7 \%$ de los casos en el rango de error Bajo y un 0,0\% en el rango de error Muy Alto. En el otro extremo, la Precisión Espacial tiene solo un 54,3\% en el rango de error Bajo y un 10,5\% en el rango de error Muy Alto. Estos valores indican que la Precisión Espacial tienen una diferencia con el Número de Isocurvas de 33,5\% en el rango Bajo y de un 100,0\% en el rango Muy Alto. En una posición intermedia, aunque más cercana al Número de isocurvas, se encuentra el error en la estimación del
Tamaño de las isocurvas, variables que se encuentran a solo un 8,4\% en el rango de error Bajo y a un 100,0\% en el rango Muy Alto, aunque este último valor debe considerar que la diferencia es de solo 3,3 puntos porcentuales (Tabla 10).

El plano cartesiano que hemos utilizado para medir la distancia entre las distintas posibilidades del muestreo y características de los sitios, de hecho, nos permite observar como todas estas variables en conjuntos producen condiciones mas o menos adecuadas para la representación de los sitios por medio de la aplicación de sondeos. En la Figura 9 es posible observar que mientras el muestreo Sistemático Radial y la fracción de muestreo de $0,25 \%$ producirán las mejores representaciones, especialmente de los sitios con una sola concentración (SitioSimple)en los cuales se representarán correctamente el número de isocurvas. En el otro extremo, el muestreo Sistemático No Alineado y las fracciones de $0,04 \%$ y $0,06 \%$ resultan las menos adecuadas, especialmente en los sitios con más de una concentración separadas (Sitio Complejo 1) y con respecto a la precisión espacial de las isocurvas. El resto de las variables se encuentran en una posición intermedia, aunque es necesario destacar que la fracción de $0,11 \%$ y la variable Tamaño se encuentra mucho más cerca del grupo de mejores condiciones para la caracterización de los sitios.

\section{Discusión}

El ejercicio aquí presentado creemos ayuda a pensar con más antecedentes la tarea de diseñar la metodología para caracterizar un sitio arqueológico con evidencias subsuperficiales de la densidad de restos arqueológicos por medio de sondeos. Esta evaluación pretende poner énfasis en cuales estrategias de muestreo probabilístico serán más certeras para caracterizar la distribución de restos depositados, lo que permite formar mejores criterios al momento de responder preguntas como ¿cuánto, cómo y dónde excavar? (Gallardo 1984), tanto como para recolectar una muestra representativa del contexto arqueológico del sitio que sirva a nuestros problemas de investigación.

En cualquier caso, es evidente que esta simulación tiene algunas limitaciones. Por un lado, las conclusiones a que aquí se ha arribado si bien son independientes del tamaño y la forma de la intervención subsuperficial utilizada, ya sea un pozo de sondeo de $1 \mathrm{x} 1 \mathrm{~m}$ o de solo unos cm de diámetro (p.ej., Hoffman 1993), como ya se señaló en el caso de los depósitos con muy baja densidad, es evidente que tamaños muy pequeños del sondeo pueden afectar las conclusiones. Por otro lado, los sitios virtuales utilizados sólo representan una de las variables que definen los sitios arqueológicos con restos depositados subsuperficialmente, la densidad de restos, excluyéndose de este análisis características de 
gran importancia como la disposición estratigráfica, la profundidad de los depósitos o la presencia de rasgos dentro del cuerpo estratificado. De esta manera la utilidad de estas conclusiones será de mucho mayor valor para el/ la arqueólogo/a que se enfrenta a estudiar la dispersión de basuras secundarias, que respecto la presencia de rasgos (p.ej., sepulturas, fogones, estructuras, etc.) que se encuentren dentro del cuerpo estratificado (p.ej., Shott 1987). En este último caso, de hecho, es posible asumir que incluso la mejor aplicación de sondeos será inadecuada para predecir la distribución de rasgos en el sitio, al menos que estos se realicen en una alta frecuencia y se controle una de las variables que no hemos aquí considerado, el tamaño de las unidades de sondeo. Aumentar el efecto de borde de la intervención, con perímetros mayores, incrementa significativamente la posibilidad de registrar rasgos específicos, los cuales de hecho serán más o menos detectables según su propio tamaño.

Agradecimientos. Este trabajo se ha inspirado en las múltiples sesiones de trabajo en que participé con el equipo de arqueólogos de la Secretaría Técnica del Consejo de Monumentos Nacionales entre 1995 y 2016. Lorena Sanhueza hizo una revisión del manuscrito final. Se agradece los comentarios hecho por los evaluadores.

\section{Referencias Citadas}

Berenguer, J. 1984. Problemas con la definición de sitio arqueológico. En Arqueología y Ciencia. Segundas Jornadas, editado por F. Gallardo, L. Suarez y L. Cornejo, pp. 61-80. Museo de Historia Natural, Santiago.

Burger, O., L Todd, P. Burnett, T. Stohlgren y D. Stephens 2004. Multiscale and nested-intensity sampling techniques for archaeological survey. Journal of Field Archaeology 29 (3-4):409-423.

Chartkoff, J. 1978. Transect interval sampling in forest. American Antiquity 43 (1):46-53.

Contreras, D. y J. Meadows 2014. Summed radiocarbon calibrations as a population proxy: a critical evaluation using a realistic simulation approach. Journal of Archaeological Science 52:591-608.

Cook, R. y J. Burks 2011. Determining site size and structure: A fort ancient example. American Antiquity 76 (1):145-162.

Cornejo, L., F. Gallardo y B. Cases 1991. Estrategias de muestreo para la recolección superficial en sitios arqueológicos. Actas del XI Congreso Nacional de Arqueología, Tomo 1, pp. 153-168. Sociedad Chilena de Arqueología, Santiago.

Drennan, R.D.yC.E. Peterson 2011. Methods for regional demographic analysis. En Settlement Patterns in the Chifeng Region, editado por Chifeng International Collaborative Archaeological Research Project, pp. 57-79. Center for Comparative Archaeology, University of Pittsburgh, Pittsburgh.

Falabella, F., L. Cornejo, I. Correa y L. Sanhueza 2014. Organización espacial durante el período Alfarero Temprano en Chile Central: un estudio a nivel de la localidad. En Distribución Espacial en Sociedades no Aldeanas: del Registro Arqueológico a la Interpretación Social, editado por Falabella, F., L. Sanhueza, L. Cornejo e I. Correa, pp. 5188. Serie Monográfica de la Sociedad Chilena de Arqueología Nơ4, Santiago.

Félix, C. y F. Greco 1968. Teoría de Errores de Mediciones. Editorial Universitaria, Buenos Aires.

Gallardo, F. 1984. ¿Dónde, cuanto y como excavar?: acerca del diseño de excavación. Arqueología y Ciencia. Segundas Jornadas, editado por Gallardo, F., L. Suerez y L. Cornejo, pp. 81-119. Museo Nacional de Historia Natural. Santiago.

Gallardo, F. y L. Cornejo 1986. El diseño de la prospección arqueológica: un caso de estudio. Chungara Revista de Antropología Chilena 16-17:409-420.
Hoffman, C. 1993. Close-Interval core sampling: Tests of a nethod for predicting internal site structure. Journal of Field Archaeology 20 (4):461-473.

Howell, T. 1993. Evaluating the utility of auger testing as a predictor of subsurface artifact density. Journal of Field Archaeology 20 (4):475-484.

Kintigh, K. 1988. The effectiveness of subsurface testing: A simulation approach. American Antiquity 53 (4):686-707.

McCartney, P. y M. Glass 1990. Simulation models and the interpretation of archaeological diversity. American Antiquity 55 (3):521-536.

McManamon, F. 1984. Discovering sites unseen. En Advances in Archaeological Method and Theory, editado por M. Schiffer, vol. 7, pp. 223-292. Academic Press, New York.

Muller, J. 1974. The Use of Sampling in the Archaeologicas Survey. Memoirs for the Society for American Archaeology 28, Washington DC.

Nance, J. 1979. Regional subsampling and statistical inference in forested habitats. American Antiquity 44 (1):172-176.

Nance, J. y B. Ball 1986. No surprises? The reliability and validity of test pit sampling. American Antiquity 51 (3):457-483.

Neff, H., R. Bishop y E. Sayre 1988. A simulation approach to the problem of tempering in compositional studies of archaeological ceramics. Journal of Archaeological Science 15:159-172.

Orton, C. 1982. Computer simulation experiments to assess the performance of measures of quantity of pottery. World Archaeology $14(1): 1-20$.

Plog, S. 1976. Relative eficiences of sampling tetchniques for the archaeological surveys. En The Early Mesoamerica Villages, editado por K. Flannery, pp. 136-158. Academic Press, New York.

Schiffer, M., A Sullivan y T. Klinger 1978. The design of the archaeological surveys. World Archaeology 10 (1):1-28.

Shott, M. 1985. Shovel-test sampling as a site discovery technique: A case study from Michigan. Journal of Field Archaeology 12 (4):457-468.

Shott, M. 1987. Feature discovery and the sampling requirements of archaeological evaluations. Journal of Field Archaeology 14 (3):359-371. 
Sundstrom, L. 1993 A simple mathematical procedure for estimating the adequacy of site survey strategies. Journal of Field Archaeology $20(1): 91-96$.

Taylor, J. 1997. An Introduction to Error Analysis. The Study of Uncertainties in Physical Measurement. University Science Books, Sausalito.

Thomas, D. 1978. The awful truth about statistics in archaeology. American Antiquity 43 (2):231-244.
Way, A. 2017. Test-pitting and the detection of sub-surface sites: an example from Lake George, NSW. Australian Archaeology $83(1-2): 32-41$

Yorston, R., V. Gaffney y P. Reynolds 1990. Simulation of artefact movement due to cultivation. Journal of Archaeological Science $17: 67-83$.

Zavoianu, I. 1985. Morphometry of Drainage Bassins. Elsavier, Ámsterdam.

\section{Notas}

${ }^{1}$ Las simulaciones se realizaron en el sistema de información geográfica QGIS versión 2.18.6.

2 Estas fracciones se calculan en base a la intensidad del sondeo, la cual define un polígono cuyos lados son iguales a la intensidad y está subdividido en unidades de $1 \mathrm{~m}$. Cada uno de estos polígonos es muestreado por un sondeo virtual, resultando la fracción de la muestra de la proporción que el sondeo representa de la superficie del polígono. Así, la intensidad de 20 metros representa un polígono de $400 \mathrm{~m}^{2}$ el cual es muestreado con un sondeo que representa el 0,25 $\%$ de todos los posibles metros cuadrados dentro de ese polígono.

${ }^{3}$ A partir de la tabla de datos se generó una capa ráster por interpolación triangular con el método cúbico basada en la densidad estimada de cada sondeo virtual y a partir de este ráster se realizó una extracción de curvas de nivel como una capa de líneas shp. Por último, esta capa de líneas fue transformada en polígonos que definen cada curva de densidad a la escala de 1 artefacto/litro.

${ }^{4}$ No se obtuvo la isocurva 0 artefactos/litro, ya que este valor no puede ser estimado como una línea ya que todo el espacio que rodea el sitio más allá del límite de 0 artefactos/ litro tiene el mismo valor.

${ }^{5}$ Por ejemplo la distancia entre el real y el obtenido por un ensayo de $15 \mathrm{~m}$ en el lado sur del eje cardinal norte-sur será más grande que en el lado norte del mismo eje, ya que existe un aumento en la coordenada Norte derivada del hecho que está más lejos del datum y no implica una diferencia de magnitud. 